\title{
MHD and Plasma Interpretation of a Prominence Eruption Observed by SOHO
}

\author{
P.C.H. Martens \\ ESA Space Science Department, SOHO Experiment Operations Facility \\ Code 682.3, GSFC, Greenbelt, MD 20771, USA
}

\begin{abstract}
Data on the May 1, 1996 prominence eruption, jointly observed by CDS, SUMER, and EIT, aboard SOHO, Yohkoh-SXT, and Kitt Peak and Meudon observatories are analyzed to obtain information on the plasma temperature, density, and velocities, as well as the magnetic field topology and strength. It is found that the 'standard' model of an erupting helical flux tube probably applies, although questions arise on the MHD stability of the prominence flux tube. The prominence is observed to remain relatively cool during its eruption $\left(\leq 5 \times 10^{5} \mathrm{~K}\right)$, while the density and velocity vary considerably on scales down to the limit of resolution. It is found that the high density, high velocity plasma blobs can be contained by the relatively weak magnetic field, as is indeed observed.
\end{abstract}

\section{Introduction}

In May and June 1996 several filaments/prominences were observed in an international campaign among SOHO, Yohkoh-SXT, and a whole array of ground based observatories in Eurasia: Pic du Midi, Meudon, Debrecen, Wroclaw, Ondrejov, Izmiran, Irkutsk, Crimea, Ratan, and Hida. For SOHO the observational sequences are written up as Joint Observations Programme 12 (JOP012; see http://sohowww.nascom.nasa.gov/ soc/JOPs/), with data from CDS, SUMER, EIT, LASCO, and MDI. This data set is probably the most comprehensive ever obtained for filaments/prominences.

In this review I will concentrate on the best event from these observations, curiously enough a test-run of the program on May 1, 1996. Observations were made by SUMER, CDS, EIT, and LASCO aboard SOHO, Yohkoh-SXT, and on the ground by the Observatoire de Meudon, and Kitt Peak National Solar Observatory.

I will present observations of an erupting filament on the limb, and describe the conclusions that can be drawn from them in terms of topology, density, temperature, motions, and magnetic field strength and stability. The novel aspect of these observations is the clear delimitation on plasma parameters through the high resolution spatial and spectral images of CDS and SUMER. These results indicate the presence of fine structure at and below the limit of resolution, with a wide variety in densities and velocities, in an otherwise uniformly cool prominence. 


\section{Time Line of the Prominence Eruption}

SUMER and CDS obtained excellent spectroscopic data of the eruption of a filament from the SE limb on May 1, 1996, between 07 and 09 UT. These data have been described in detail by Wiik et al. (1997) and by Kucera et al. (1998, these proceedings). Experience during later runs of JOP012 has shown that it is extremely difficult to pinpoint the exact time and location of a filament eruption and, therefore, the data from the May 1 test run have become extremely valuable.

In this review the CDS/SUMER spectroscopic data will be analyzed in a wider context. Using available magnetograms, UV, and soft X-ray data for April 30 , and May 1, I have compiled the time line in Table 1 which summarizes the main events and data availability.

Table 1

Time Line for the May 1, 1996, Prominence Eruption and CME

Time

30 April, 10:05 UT

30 April, 21:21 UT

and onward

30 April

14:10-15:05 UT

1 May, 01:17 UT \& 01:29 UT

1 May, 07:21 UT

$1 \mathrm{May}, 07: 25 \mathrm{UT}$

1 May, 07:13-09:55 UT

1 May, 10:00-18:00 UT
Event/Observation

Yohkoh-SXT Image with a Clear

Dark Filament Channel and

Off-limb Keyhole

High Prominence on SE Limb in EIT $304 \AA$

Kitt Peak Magnetogram, with EW Polarity Inversion Line at SE Limb

EIT 284 and $195 \AA$ Images show Filament Channel but no Keyhole

Filament Channel in Yohkoh-SXT has straightened

Meudon CA II K3 Spectroheliogram, with CDS (box) and SUMER FOV (crosses in CDS box)

Spectroscopy of Filament Eruption by CDS and SUMER

LASCO C3 Observations of CME from $\mathrm{E}$ Limb
Reference

Figure 1a

Figure 2

Figure 1b

Figs. 1c \& 1d

Figure 3a

Figure $3 b$

see Kucera et al. these proc., and Wiik et al. 1997

Wiik et al. 1997 

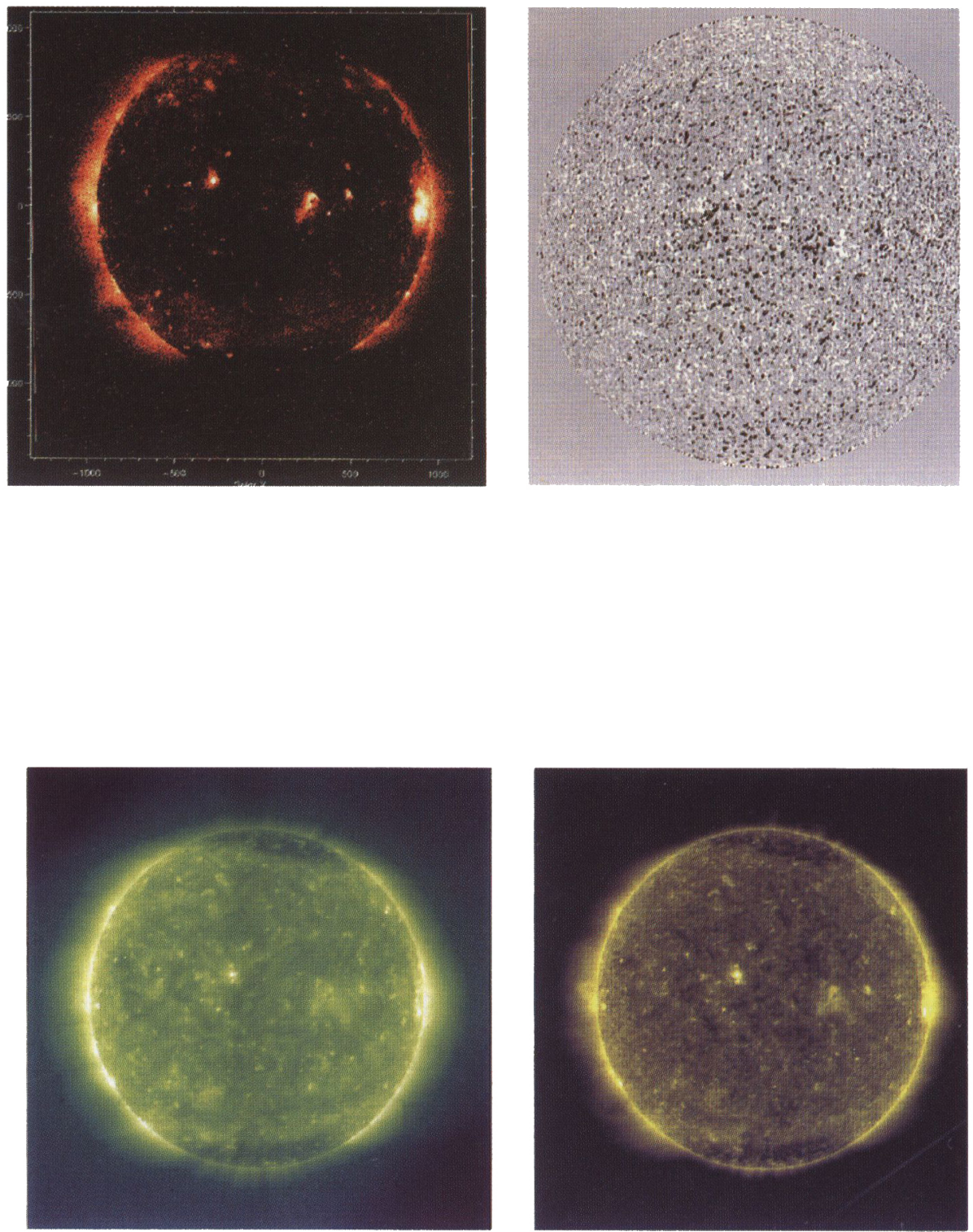

Figure 1. Four full disk images on April 30, 1996. Clockwise from top left: (a) Yohkoh-SXT at 10:05 UT, (b) Kitt Peak Magnetogram at 14:10 UT, (c) SOHO-EIT, $284 \AA$, at 01:17 UT, and (d) SOHO-EIT, 195 $\AA$, at 01:29 UT. Note the polar coronal hole polarity inversion line and filament channel in the SE quadrant, and the "keyhole" in the SXT image, at the east extension of the filament channel. 


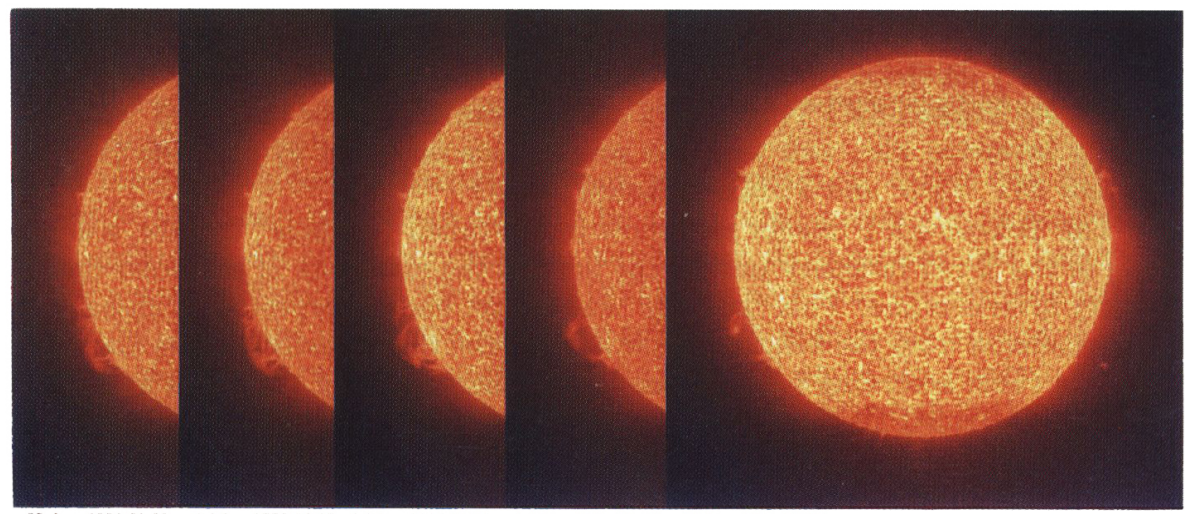

30-Apr-19\% 21:21 1-May-19\% 02:13 1-May-19\% 11:06 1-May-1996 19:45 2-May-1996 03:23

Figure 2. A time series of EIT $304 \AA$ observations of the development of a prominence on the SE limb. The EIT $304 \AA$ images consist of a blend of He II and Si XI line radiation; for prominences this is dominated by $\mathrm{He}$ II at about $60,000 \mathrm{~K}$.
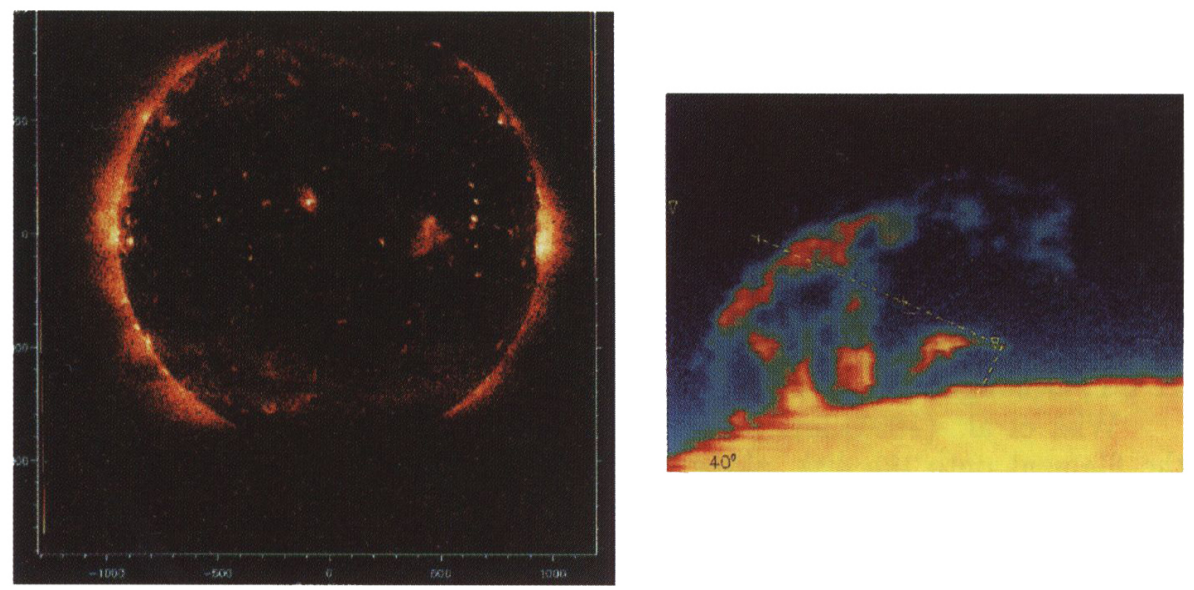

Figure 3. Left (a): A Yohkoh-SXT full disk image on May 1, 07:21 UT, during the partial prominence eruption on the SE limb. Right (b): Meudon Ca II K3 Spectroheliogram, taken at 07:25 UT, with CDS field of view delineated by the box and SUMER by the crosses. 


\section{Plasma Environment: Interpretation}

The Kitt Peak full-disk magnetogram (Figure 1a) demonstrates an absence of large-scale organized magnetic features, except for the polar coronal hole magnetic polarity inversion lines, both north and south. The dark prominence channel seen by Yohkoh-SXT (Figures 1a and 3a) can clearly be identified with the polarity inversion line, and with the prominence observed by Meudon (Figure $3 \mathrm{~b})$, and EIT, CDS, and SUMER aboard SOHO.

The Yohkoh-SXT images also show the so-called "keyhole" feature, a darkening in the X-ray intensity just off the limb at the extension of the polar crown polarity inversion line. This "keyhole" has always been interpreted as absence of $\mathrm{X}$-ray emission from the helical magnetic flux tube that constitutes the filament. This flux tube, according to many models for filaments (e.g., Martens and Kuin 1989), is suspended above the polarity inversion line, with its axis parallel to it. Thus, just off the limb, where the axis is parallel to the line-of-sight, one would indeed notice an absence of soft X-ray emission, in the form of a cross-section of the filament flux tube.

This interpretation is supported here by the clearly visible filament channel on the disk. The Yohkoh-SXT observations for this filament thus suggest a relatively "cool" filament, with temperatures less than 1 million $\mathrm{K}$. The SUMER and CDS observations below will confirm this. "Cool" filament channels are relatively rare among the Yohkoh-SXT observations of filaments; more often one sees the "spine" of the filament quite clearly in soft X-rays, in particular just before and during an eruption. What causes the absence of soft X-ray emission in this case, is not known.

The height above the limb of the prominence at the onset of the eruption $(\approx$ $07 \mathrm{UT}$ ) is $100,000 \mathrm{~km}$, very high in comparison with most other filament eruptions. In the Martens and Kuin model (1989) this requires that the photospheric magnetic field below the filament is uniform over about the same scale-size. This is consistent with the Kitt Peak magnetogram.

The SUMER and CDS observation show that the middle part of the prominence erupts, well within their field of view. Both instruments see an expanding loop that heats up somewhat (from $100,000 \mathrm{~K}$ to just under $500,000 \mathrm{~K}$ ), but no soft X-ray emitting plasma is produced, as Yohkoh-SXT observations confirm. The EIT data (see Figure 2) demonstrate that the prominence has reformed by 11 UT, although its detailed structure is quite different.

Wiik et al. (1997) interpret the most southern leg as the continuation of the filament onto the disk, along the filament channel. There is also a small loop between the southern legs, which in the CDS and SUMER observations is seen to be the one that erupts. These observations exclude the possibility of a thermal DB, since the loop is clearly seen moving upward in subsequent images and remains visible in line spectra. Thus, it appears that only the middle part of the prominence, with its continuation onto the disk, erupts and reforms very quickly.

The fact that the prominence reforms so quickly - it is observed by EIT at 11:06 UT in $\mathrm{He} \mathrm{II} / \mathrm{Si} \mathrm{XI}$ at $304 \AA$, at a temperature of about $60,000 \mathrm{~K}$, and also by CDS in a much smaller field-of-view at 11:00 UT - makes one wonder whether the prominence middle section really erupted. Is it possible that what 
we are observing is merely the disconnection and retraction of one of the feet of the prominence, the inner southern one at about $533^{\circ}$ ? The retraction of the feet of a prominence during its evolution is a well known phenomenon from $\mathrm{H} \alpha$ movies of prominences. However, the simultaneous occurrence of a $\mathrm{CME}$ observed by LASCO at about the same position angle (see Wiik et al., for a detailed description) might be taken as an indication of a real eruption, although it is certainly not a sure sign.

If there was indeed a partial eruption of the filament within the field of view of SUMER and CDS, then this can be interpreted within the now more or less standard model of an erupting flux tube, with reconnection just below it; see, for example, Figure 1 in Hirayama (1974). We note that the Meudon image (Figure $3 \mathrm{~b}$ ) gives the impression of a flux tube with many coils around it, and this is reinforced by careful inspection of the SUMER and CDS rasters.

Stability against the kink mode for a flux-tube attached at both ends has been investigated by Kadomtsev (1966) for the Tokamak case, and by Hood and Priest $(1979,1981)$ for solar coronal flux tubes. Their results suggest that only one, or at most a few, turns are permitted before instability sets in; the exact number being determined by the details of the model and the boundary conditions. In the case of filaments the mass loading at the lowest points in the coils by the filament material will have a stabilizing influence, but I am not aware of any studies to quantify this. In the absence of such studies, and given the generally low plasma $\beta$ in the solar corona, one would not expect this mass loading to change the stability criteria appreciably. Hence, if we maintain the interpretation of the prominence as a helical flux tube, we are faced with the problem that this flux tube should be MHD-unstable according to our theoretical knowledge. The observations do not show the development of a kink, however. Indeed the same prominence is observed almost intact one day later by EIT (Figure 2). The same phenomenon has been observed in several other prominences by SOHO. I conclude that it is time to perform detailed MHD stability calculations for flux tubes with mass loading, and if the contradiction persists, for a reexamination of current prominence models.

The detailed analysis of the CDS and SUMER observations with respect to the plasma parameters of the prominence has been reported upon in detail by Wiik et al. (1997). Here I will just summarize the most salient conclusions:

- $\mathrm{T} \approx 2 \times 10^{4} \mathrm{~K}-5 \times 10^{5} \mathrm{~K}$ (highest in erupting part)

- $\mathrm{n} \approx 3 \times 10^{9} \mathrm{~cm}^{-3}-3 \times 10^{11} \mathrm{~cm}^{-3}$ (large dispersion)

- $\Delta \mathrm{v} \approx 100 \mathrm{~km} / \mathrm{sec}\left(\geq \mathrm{c}_{s} \approx 40 \mathrm{~km} / \mathrm{sec} \sqrt{T}_{5}\right)$

The density determinations from density-sensitive line ratios show a large pixel by pixel $(1 \mathrm{arcsec})$ variation along the slit, indicating the presence of unresolved (filamentary?) fine structure in the erupting loop. The observed Doppler shifts are predominantly red in the northern leg of the loop and blue in the other, and of the order of $40 \mathrm{~km} / \mathrm{sec}$, consistent with large scale downdrafts, as one might expect in an erupting loop. SUMER, due to its higher spectral and spatial resolution, sees velocities up to about $100 \mathrm{~km} / \mathrm{sec}$, with sometimes several structures along the line-of-sight with a large velocity dispersion. Such high velocities are unusual (Schmieder 1989). We note that for this relatively 
cool prominence the Doppler shifted velocities are of the order of, or larger than, the sound velocity (see the equation in the third bullet above) and horizontal. Hence, the motion must be driven by a magnetic force, rather than pressure or gravity. Perhaps we are seeing jets from the reconnection region below the erupting part of the prominence, as, for example, modeled by Shibata et al. (1992).

Finally, we note that for a polar crown prominence of such large height, a magnetic field strength of at most about $10 \mathrm{G}$ can be expected. This is borne out by measurements of the magnetic field in other polar crown prominences through the Hanle effect by Bommier and Leroy (1998, these proceedings, and references therein). On the other hand, the observed Doppler shifts can be interpreted as plasma motions along the helical magnetic field lines that make up the prominence. Since the prominence flux tube preserves its integrity, even while it erupts, its magnetic field must be strong enough to contain the plasma motions inside. This implies that the centrifugal force from the plasma motions must be smaller than the tension force in the flux tube, i.e.,

$$
\frac{\rho v^{2}}{2 R} \leq \frac{B^{2}}{4 \pi R}
$$

where $\rho$ is the mass density, $v$ the plasma velocity, $B$ the magnetic field strength, and $R$ the radius of the prominence flux tube. Using the maximum value for the observed particle density given above, and a plasma velocity of $100 \mathrm{~km} / \mathrm{sec}$, it is found that the magnetic field strength must be larger than $12.5 \mathrm{G}$, of the same order as the field strength expected for these types of prominences. Since the plasma velocity is of the order of, or larger than the sound velocity in this cool prominence, the plasma $\beta$ is of the order of unity for the observed density and a field of around $10 \mathrm{G}$. Hence, it appears that the high density parts of the fine structure have the highest density that can be accommodated in this type of prominence.

\section{Conclusions}

The data from the joint observations described in this paper have allowed us to derive the plasma parameters for an erupting prominence in unprecedented detail. Prominence eruptions, and the frequently accompanying flares and CMEs, are complex phenomena, involving a large scale reconfiguration of the magnetic field, with plasma effects on all scales, from giant arcades to small reconnection flows and jets. No single instrument can provide a complete data set including all these effects and, therefore, well coordinated multi-observatory campaigns, followed up by joint data analysis efforts, are necessary to enhance our understanding of prominences and their eruptions. SOHO has been the central element in a series of prominence campaigns, and its continued operations through solar maximum will provide the solar physics community with a unique opportunity for further study of prominences. The catalog of SOHO coordinated observations is available on the Web for anyone interested (http://sohodb.nascom.nasa.gov/cgibin/soho_campaign_search), and the campaign data enter the public domain after one year. 
Acknowledgments. SOHO is a mission of international collaboration between ESA and NASA. I am grateful to J. Harvey and Z. Mouradian for providing me with Kitt Peak and Meudon data shown here, to B. Thompson of EIT for preparing Figure 2, to the Yohkoh-SXT team for allowing me to reproduce their data, and to J. Wiik, B. Schmieder, T. Kucera, and the CDS and SUMER teams for letting me use freely their yet unpublished results in presenting and preparing this paper. Last but not least, I want to thank the organizers of this excellent, timely, beautifully located, and well organized colloquium for their efforts.

\section{References}

Hood, A. W and Priest, E. R. 1979, Solar Phys., 64, 303

Hood, A. W and Priest, E. R. 1981, Geophys. Astrophys. Fluid Dyn., 17, 297 Hirayama, T. 1974, Solar Phys., 34, 323

Kadomtsev, A. A. 1966, in Reviews of Plasma Physics, Vol. 2, M. A. Leontovich (ed.), Consultants Bureau: New York, p. 153

Martens, P. C. H. and Kuin, N. P. M. 1989, Solar Phys., 122, 263

Shibata, K., Nozawa, S. and Matsumoto, R. 1992, Publ. Astron. Soc. Japan, 44, 265

Schmieder, B. 1989, in Dynamics and Structure of Quiescent Prominences, E. R. Priest (ed.), Kluwer: Dordrecht, Holland, p. 15

Wiik J. E., Schmieder, B., Kucera, T., Poland, A., Brekke, P. and Simnett, G. 1997, Solar Phys., 175, 411 